\title{
Piggyback Rendezvous Cannulation for Endoscopic Metal Stent Conversion of Percutaneous Transhepatic Biliary Drainage
}

\author{
Vincent Zimmer ${ }^{1,2}$ \\ ${ }^{1}$ Department of Medicine, Marienhausklinik St. Josef Kohlhof, Neunkirchen, ${ }^{2}$ Department of Medicine II, Saarland University Medical Center, \\ Saarland University Homburg, Homburg, Germany
}

Biliary rendezvous procedures typically imply over-thewire cannulation after guidewire insertion via a percutaneous transhepatic biliary drainage (PTBD). Alternative techniques, such as the parallel cannulation method alongside the PTBD catheter have been reported. ${ }^{1}$ More recently, other escape techniques that substitute guidewires for different interventional radiology (IR) catheters have been described, such as angiography Cobra 2 and Kumpe access catheter, with the aim at retrograde cannulation after establishing appropriate end-to-end contact. ${ }^{2,3}$ However, these techniques require specialized IR accessories and/or competence. This report introduces another easy-to-perform variant technique for rendezvous procedures that resort to ubiquitously available endoscopy technology and minimal need for external (rendezvous) cooperation.

As the first step of the procedure, the indwelling 10-F internal-external PTBD drain is withdrawn under duodenoscopic control up to the papilla. Following this, the tip of a 5.5-F endoscopic retrograde cholangiopancreatography (ERCP) catheter, with a conical metal tip, is pushed into the orifice of the PTBD drain until achieving proper alignment. Subsequently, the common bile duct stricture is traversed in a piggyback fashion using coordinated pull (PTBD) and push (ERCP catheter) maneuvers. After releasing the end-

Received: July 7, 2017 Revised: August 18, 2017

Accepted: September 13, 2017

Correspondence: Vincent Zimmer

Department of Medicine, Marienhausklinik St. Josef Kohlhof, Klinikweg 1-5, Neunkirchen 66539, Germany

Tel: +49-6821-3632070, Fax: +49-6821-3632624, E-mail: vincent.zimmer@gmx.de ORCID: https://orcid.org/0000-0002-6298-4717

(c) This is an Open Access article distributed under the terms of the Creative Commons Attribution Non-Commercial License (http://creativecommons.org/ licenses/by-nc/3.0) which permits unrestricted non-commercial use, distribution, and reproduction in any medium, provided the original work is properly cited. to-end contact, the PTBD is withdrawn into the intrahepatic biliary system (provisionally left in place until the procedure is finished). In the elderly, frail patient depicted in the figure and video, after cannulation of the route with guidewire insertion, a 10- $\times 60-\mathrm{mm}$ transpapillary biliary metal stent was placed for definitive palliative treatment and the PTBD was removed (Fig. 1, Supplementary Video 1).

The potential benefits of the presented "piggyback rendezvous cannulation" technique include minimal need for percutaneous cooperation, thus obviating the need for a second endoscopist with specialized training and/or IR cooperation. Given the predominant endoscopic part of the rendezvous with minimal PTBD manipulation, other potential benefits may be discussed, such as reduced patient and staff radiation exposure, and time and equipment consumptions.

\section{Conflicts of Interest}

The author has no financial conflicts of interest.

\section{Supplementary Material}

Video 1 . The video illustrates the novel piggyback cannulation technique for rendezvous metal stent conversion of percutaneous transhepatic biliary drainage (https://doi.org/10.5946/ce.2017.110.v001).

\section{REFERENCES}

1. Dickey W. Parallel cannulation technique at ERCP rendezvous. Gastrointest Endosc 2006;63:686-687.

2. Lee TH, Park SH, Lee SH, et al. Modified rendezvous intrahepatic bile duct cannulation technique to pass a PTBD catheter in ERCP. World J Gastroenterol 2010;16:5388-5390.

3. Chang JH, Lee IS, Chun HJ, et al. Comparative study of rendezvous techniques in post-liver transplant biliary stricture. World J Gastroenterol 2012;18:5957-5964. 

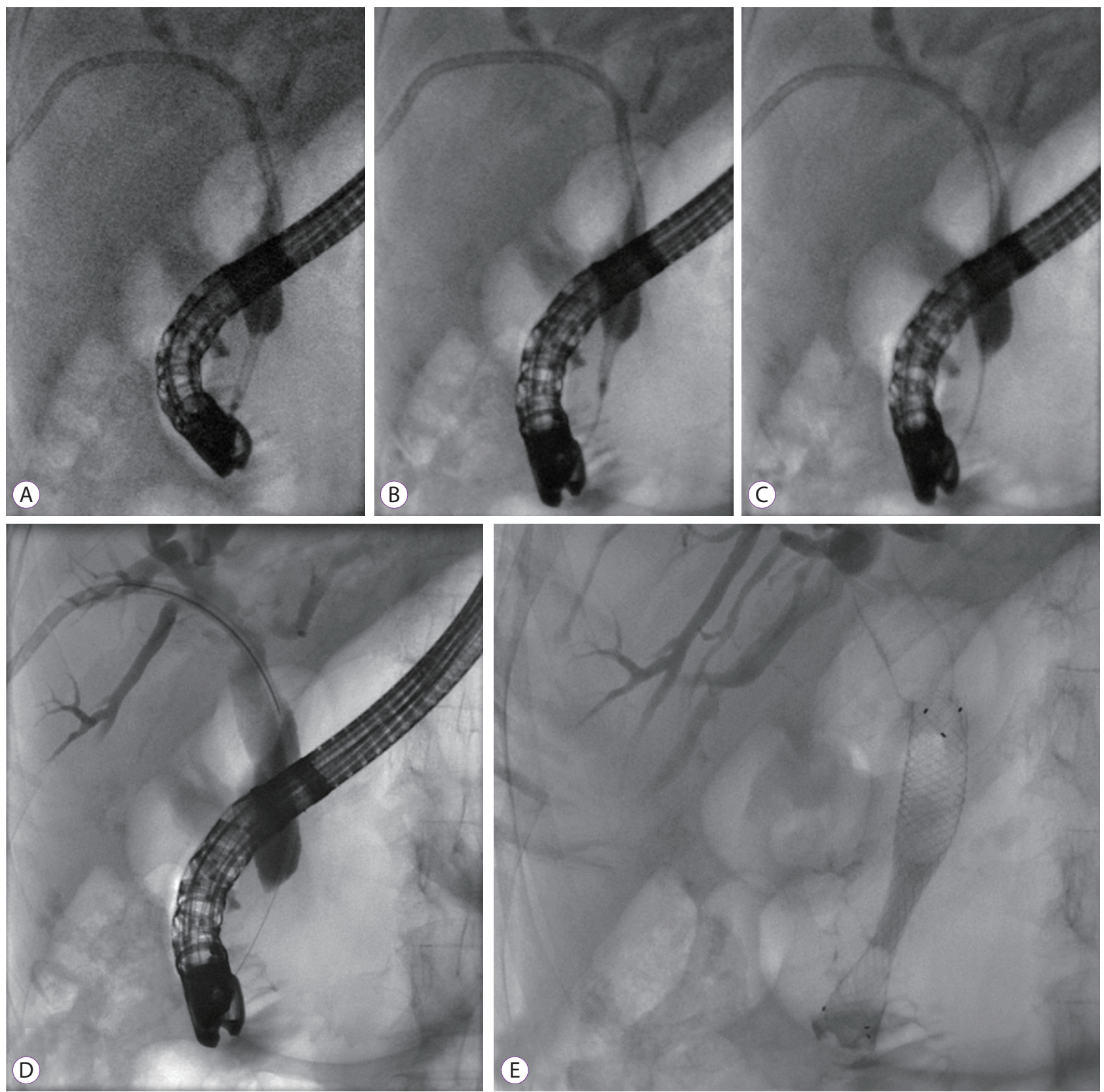

Fig. 1. Different steps of the novel piggyback technique for rendezvous cannulation with biliary metal stent conversion. (A) Cannulation of the withdrawn 10-F percutaneous transhepatic biliary drainage (PTBD) catheter with a conical, tapered-tip, 5.5-F endoscopic retrograde cholangiopancreatography (ERCP) catheter (Endoflex, Voerde, Germany). (B) Pushing of the cannula into the intern-extern drain for proper alignment (note spraining of the PTBD catheter). (C) Crossing of the common bile duct stricture using coordinated push (ERCP) and pull (PTBD catheter) maneuvers. (D) Removal of the ERCP cannula after guidewire insertion (0.035-inch Jagwire; Boston Scientific, Ratingen, Germany) and release of the end-to-end contact. (E) Final documentation of the transpapillary inserted, partially covered biliary metal stent (Leufen Medical, Aachen, Germany) after PTBD removal. 